\title{
QUININA: 470 ANOS DE HISTÓRIA, CONTROVÉRSIAS E DESENVOLVIMENTO
}

\author{
Alfredo Ricardo Marques de Oliveira* e Daiane Szczerbowski \\ Departamento de Química, Universidade Federal do Paraná, CP 19081, 81531-990 Curitiba - PR, Brasil
}

Recebido em 26/11/08; aceito em 30/3/09; publicado na web em 10/8/09

\begin{abstract}
QUININE: 470 YEARS OF HISTORY, CONTROVERSY AND SCIENCE DEVELOPMENT. After 470 years, a history of development, international seed smuggling and scientific development that caused deep changes in our society, has reached an end. In 1638, the countess of Chinchón contracted a disease while in the Amazon rain forest and was healed by a potion used by the native inhabitants. In 1856, William H. Perkin while attempting to obtain synthetic quinine, discovered the mauveína, a molecule that changed the world. The synthesis of quinine was also the subject of a bitter controversy among two excellent scientists of the $20^{\text {th }}$ century. During centuries, quinine was the only hope against malaria disease and its exploration almost extinguished the Cinchona tree.
\end{abstract}

Keywords: quinine; chemical industry; Cinchona alkaloids.

\section{CONQUISTAS, POLÍTICA E RELIGIÃO}

1638 - A história registra que neste ano, ${ }^{1}$ a condessa de Chinchón, esposa do vice-rei espanhol no Peru, foi acometida de forte febre terçã. Ao ingerir uma poção feita pelos índios chamada "quina-quina" a febre cedeu e a continuidade do tratamento a deixou curada. Este evento pode ser estabelecido como o início de uma história de desenvolvimentos, experimentações e enganos, envolvendo alguns dos maiores nomes da ciência dos últimos 470 anos.

A partir deste relato, padres jesuítas da missão espanhola levaram o pó para a Europa para vendê-lo como um medicamento, que depois ficou conhecido como “pó dos jesuítas”. Em 1679, o Rei Charles II da Inglaterra foi vitimado por uma forte febre, porém sendo protestante, preferia morrer a tomar um medicamento católico, por melhor que ele fosse. Neste contexto, surge Robert Talbor com um medicamento "protestante" que o rei não hesitou em tomar. Ficou curado e como agradecimento sagrou Talbor, cavaleiro e médico real. Alguns anos depois foi revelado que o remédio protestante de Talbor era na verdade o "pó dos jesuítas" apenas em uma formulação diferente.

Hoje sabemos que o mal que afligiu a condessa e o rei, era a malária. Este nome tem origem na expressão italiana "mala aria" (ar ruim),${ }^{2}$ pois se acreditava que a doença era transmitida pelo ar contaminado proveniente de pântanos e esgotos. Na verdade a malária é causada pelo protozoário Plasmodium falciparum, descrito em 1880 pelo médico francês Charles Louis Alphonse Laveran, sendo transmitida pela picada das fêmeas do mosquito do gênero Anopheles. ${ }^{3}$

A árvore de Cinchona tem cerca de $20 \mathrm{~m}$ de altura, pertence à família das Rubiáceas, que possui entre outros membros, o café e as gardênias. Os europeus, em homenagem à condessa Chinchón, classificaram o gênero como Cinchona do qual as espécies mais importantes são: Cinchona ledgeriana, C. officinalis, C. calisaya e C. pubescens .

Até 1820, apenas um pó feito com as raízes da árvore era comercializado. Nesse ano, Pelletier e Caventou isolaram deste pó, ${ }^{4}$ um alcaloide com extrema atividade contra a malária, ao qual deram o nome de quinina. Após a descoberta, inúmeros métodos foram desenvolvidos para extrair o alcaloide e vendê-lo como medicamento. A extração e exportação para a Europa era um processo tão lucrativo que o governo peruano proibiu a exportação de semente de Kina (nome indígena da árvore) para manter o controle sobre este

*e-mail: armo@quimica.ufpr.br mercado. No entanto, ingleses e alemães contrabandearam algumas sementes e formaram novas plantações: os alemães na ilha de Java, Indonésia e os ingleses na Índia e no Ceilão (Sri Lanka). Para azar dos contrabandistas, as sementes contrabandeadas não eram das espécies que possuíam as maiores porcentagens de quinina ( $C$. calisaya pelos alemães e de $C$. pubescens pelos ingleses) e assim a extração das árvores amazônicas continuou. Finalmente, os alemães compraram por US\$20,00 uma libra de sementes $(453,592 \mathrm{~g}) \mathrm{de}$ Cinchona ledgeriana, que possui a maior porcentagem de quinina e rapidamente estabeleceram plantações extensivas desta espécie, dominando o mercado mundial. Em 1918, a maior parte do mercado mundial era suprido pelos alemães que obtiveram enormes lucros com este comércio, enquanto o Peru e a Bolívia, de onde o medicamento foi originado, quase nada receberam.

\section{QUININA: A MOLÉCULA REVELADA}

A extração da quinina a partir da casca de Cinchona não rende tanto quanto a extração a partir da árvore inteira. Assim, a obtenção comercial da quinina quase levou à extinção as árvores amazônicas, as quais, ironicamente foram replantadas a partir de sementes obtidas das mesmas plantações formadas pelo contrabando.

A necessidade de obter a quinina de maneira mais fácil e mais barata deu um grande impulso para o desenvolvimento da ciência e da indústria química, tal como a conhecemos. Após o isolamento por Pelletier e Caventou em 1820, ${ }^{4}$ Louis Pasteur em 1852 observou que a molécula era levorrotatória,${ }^{5}$ porém nesta época ainda não se tinha nem o conhecimento da fórmula molecular e nem da estrutura espacial da quinina. Em 1854, Strecker determinou que a fórmula molecular da quinina era $\mathrm{C}_{20} \mathrm{H}_{24} \mathrm{~N}_{2} \mathrm{O}_{2} \cdot{ }^{6}$

\section{A HISTÓRIA SE DIVIDE!}

Neste ponto a história bifurca: William Henry Perkin, ${ }^{7}$ aos 18 anos, fez a primeira tentativa de obter a quinina sintética. No seu pequeno laboratório montado em casa, Perkin tentou obter a quinina a partir da oxidação da aliltoluidina (1), cuja fórmula molecular é $\mathrm{C}_{10} \mathrm{H}_{13} \mathrm{~N}$. Uma vez que a aliltoluidina (1) tinha todos os elementos químicos necessários a menos de duas moléculas de água, Perkin tentou obter a quinina pela dimerização oxidativa da aliltoluidina (1). Isto demonstra bem o parco domínio da química na época (Figura 1). 


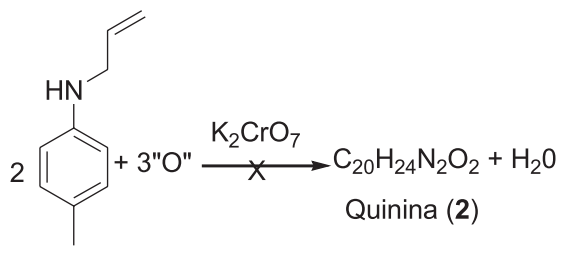

Figura 1. Primeira tentativa para sintetizar a quinina (2) em 1856

O produto obtido foi um precipitado escuro, "grudento" e intratável! Para simplificar o experimento, Perkin substituiu a aliltoluidina (1) pelo sulfato de anilina e repetiu a oxidação, ${ }^{8}$ obtendo mais uma vez um precipitado escuro. No entanto, a extração deste novo precipitado com etanol deu origem a uma solução de cor púrpura, profunda e consistente, que ele chamou de Púrpura Tiriana, por referência à antiga púrpura extraída em Tiro, e os franceses chamaram de mauve.

Perkin em seguida fez alguns testes com o tingimento de papéis e tecidos e fundou uma pequena fábrica para produzir o corante para as fábricas de tecidos, então em plena revolução industrial. Isto deu origem à moderna indústria química de corantes, perfumaria e medicamentos. Sua descoberta influenciou o destino de várias empresas, principalmente a BASF (Badische Anilin-\& Soda-Fabrik), AGFA (Aktiengesellschaft für Anilinfabrikation) Bayer e Hoechst, que diversificaram sua produção em vários outros segmentos importantes da indústria química. Perkin recebeu o título de Sir em 1906 e morreu em 1907 de causa desconhecida.

Na verdade só foi possível elucidar a estrutura química da mauveína em 1994, utilizando técnicas modernas de purificação e análise estrutural. Meth-Cohn purificou e analisou uma amostra do corante originalmente feito por Perkin, ${ }^{9,10}$ isolando dois compostos diferentes: a mauveína A e mauveína B. Em 2007, Seixas de Melo e colaboradores reproduziram a síntese de Perkin e isolaram dois outros novos compostos: mauveína B2 e mauveína $C,{ }^{11}$ os quais estavam também presentes em uma amostra original obtida no Museu de Ciências de Londres (Figura 2).<smiles>Cc1ccc(Nc2ccc3nc4cc(C)c(N)cc4[n+](-c4ccccc4)c3c2)cc1</smiles><smiles>Cc1ccc(Nc2cc(C)c3nc4cc(C)c(N)cc4[n+](-c4ccccc4)c3c2)cc1</smiles><smiles>Cc1ccc(Nc2ccc3nc4cc(C)c(N)cc4[n+](-c4ccc(C)cc4)c3c2)cc1</smiles>

Mauveína B2<smiles>Cc1ccc(Nc2cc(C)c3nc4cc(C)c(N)cc4[n+](-c4ccc(C)cc4)c3c2)cc1</smiles>

Mauveína C

Figura 2. Estruturas identificadas em 1994 e 2007

\section{SÍNTESE DA QUININA: UM MITO?}

O segundo ramo da bifurcação nos traz de volta à síntese da quinina. Entre 1907-09, Paul Rabe determinou a conectividade correta entre os átomos da quinina (2), ${ }^{12}$ no entanto, a determinação da estereoquímica absoluta teve que esperar até 1967 quando foi possível a elucidação por cristalografia de raios-X (Figura 3). ${ }^{13}$

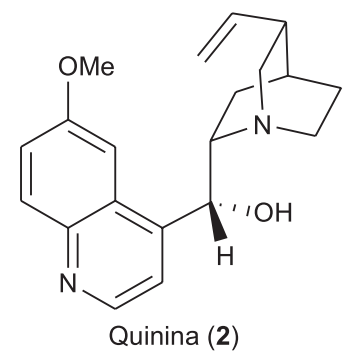

Figura 3. Estrutura atribuída a quinina (2)

Em 1853, Louis Pasteur, fez o tratamento da quinina (2) com ácido sulfúrico e isolou a $d$-quinotoxina (3) (Figura 4). ${ }^{14}$

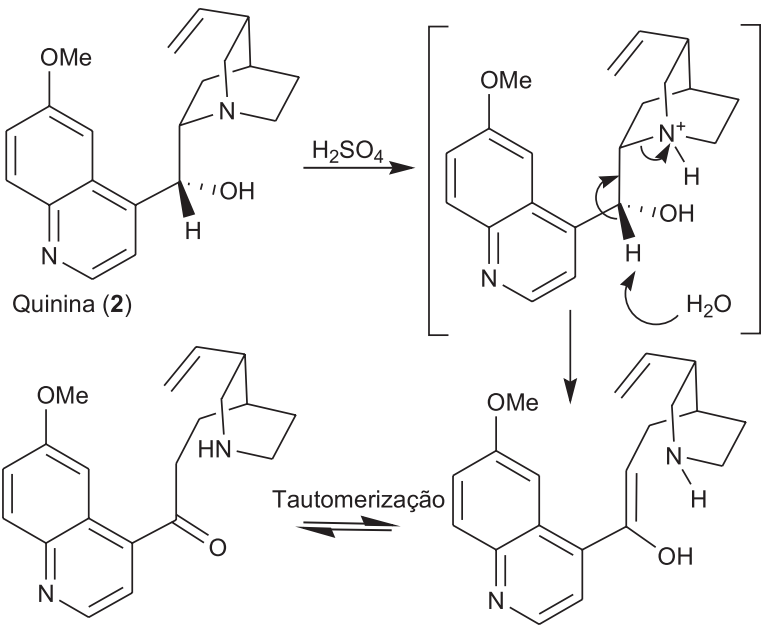

Quinotoxina (3)

Figura 4. d-quinotoxina (3) obtida em 1853

Em 1918, Paul Rabe e Karl Kindler publicaram um artigo intitulado "Partial Synthesis of Quinine. The Cinchone Alkaloids. XIX" ${ }^{15}$ onde descreveram, com poucos detalhes experimentais, a transformação inversa àquela realizada por Pasteur. Ou seja: partindo da $d$-quinotoxina (3), obtiveram a $\alpha$-halocetona (4), que foi transformada na quininona (5), a qual foi reduzida formando a quinina (2) (Figura 5). Em 1939, Rabe recuperou uma amostra do trabalho de 1918, recristalizou e caracterizou mais uma vez a quinina (2), obtida pelo método acima.

No entanto, com a invasão da Polônia em 1939 e o início das hostilidades que levaram à Segunda Guerra Mundial, havia grande interesse na obtenção da quinina (2) sintética, uma vez que as plantações comerciais estavam localizadas na ilha de Java na Indonésia, então sob domínio japonês.

Neste contexto de guerra, Robert Burns Woodward, tido como um dos "pais" da síntese orgânica, publicou em 1944 um trabalho com o título "Total Synthesis of Quinine" em colaboração com William von Eggers Doering. ${ }^{16}$ Este trabalho, apenas uma comunicação inicial, foi seguido por uma publicação completa em 1945 e deu origem a uma das grandes controvérsias do século XX. ${ }^{17} \mathrm{Nas}$ duas publicações, Woodward e Doering recorreram ao trabalho de Rabe e Kindler para afirmar que haviam sintetizado a quinina (2). $\mathrm{Na}$ verdade, o que Woodward e Doering obtiveram foi a síntese do homomeroquineno (6) racêmico e também a quinotoxina (3) (Figura 6). Uma vez que Rabe já havia transformado a quinotoxina (3) na quinina (2), Woodward aparentemente acreditou ser suficiente a referência ao trabalho de Rabe e Kindler, no que hoje seria conhecido como uma síntese formal. 


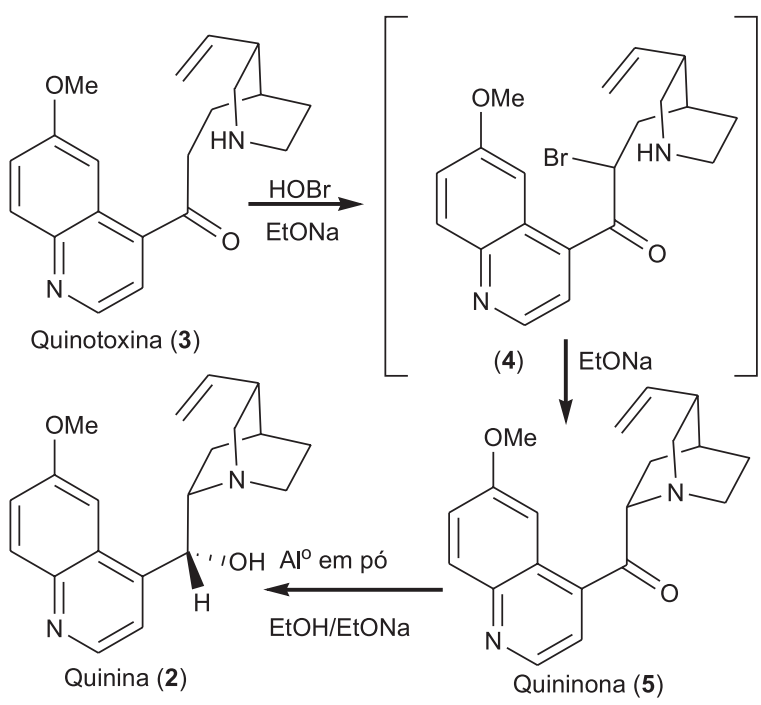

Figura 5. Síntese parcial da quinina (2) em 1918
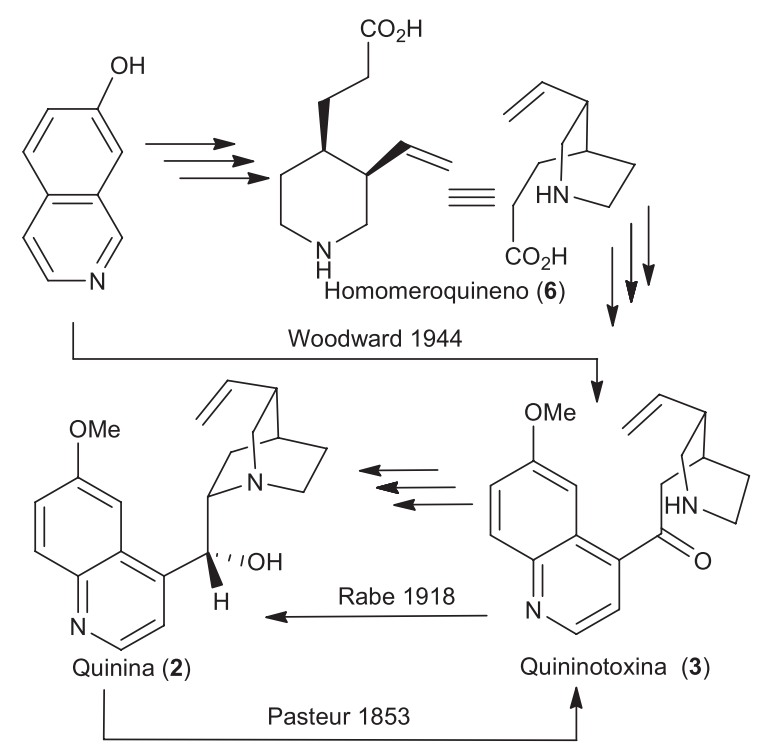

Figura 6. Síntese do homomeroquineno (6) e da quinotoxina (3)

Porém em 1944, Gilbert Stork, também um dos grandes nomes da síntese orgânica, escreveu a Woodward perguntando se ele havia reproduzido o trabalho de Rabe e Kindler para a obtenção da quinina (2). Não obteve resposta. Em 2000, Stork declarou em uma entrevista a $C \& E N N e w s,{ }^{18}$ que a síntese da quinina (2) por Woodward em 1944 não passava de um "mito acreditado por muitos". Em 2001, Stork publicou um artigo intitulado "The First Stereoselective Synthesis of Quinine", ${ }^{19}$ onde, a partir de um único estereocentro definido no material de partida, todos os outros estereocentros foram construídos. Neste trabalho, Stork comenta que "Eram tempos de guerra. Os Estados Unidos haviam sido expulsos da Indonésia e assim perdido acesso às raízes de cinchona. Esta ansiedade pode explicar as notícias e um notável entusiasmo no lugar de uma análise sóbria e racional, a qual criou a impressão "quasiuniversal" de que a construção do homomeroquineno, significava que a quinina havia sido sintetizada. Notavelmente, a confusão promovida por este e por centenas de outros artigos contemporâneos persistiu até os dias de hoje".

Em 2007, Seeman publicou uma revisão no Angewandte Chemie,${ }^{20}$ analisando não apenas a controvérsia criada em torno da síntese da quinina (2) mas também os homens envolvidos, sua ética e a credibilidade dos resultados publicados. Assim, observando a história e entrevistando alguns dos personagens envolvidos ainda em atividade, Seeman pode deduzir que Woodward e Doering ${ }^{21}$ haviam sim obtido o homomeroquineno (6) bem como a quinotoxina (3) e que apenas não enxergaram razões para repetir o trabalho de Rabe e Kindler. Tendo em mente que em 1939 Rabe recuperou uma amostra feita em 1918, a recristalizou e caracterizou não há porque duvidar que a quinina (2) não tenha sido parcialmente sintetizada em 1918.

Em janeiro de 2008, Smith e Williams repetiram o trabalho de Rabe e Kindler, ${ }^{22}$ apresentando uma última surpresa: na última etapa executada por Rabe em 1918, está descrita a redução com alumínio em pó. Em 2008, 90 anos depois, esta reação não forneceu o resultado esperado. Smith e Williams então pensaram que tipo de alumínio um químico do início do século XX teria usado? Certamente não seria um alumínio extra puro adquirido de uma companhia especializada. Fizeram então a mesma reação com alumínio "aerado" e o resultado obtido foi a síntese da quinina (2) como descrito por Rabe e Kindler em 1918.

Assim, em 2008, 470 anos depois de iniciada, chegou ao fim a história registrada desta molécula, que foi importante para o ciclo de colonização europeu e esteve envolvida em disputas religiosas; sua descoberta ocasionou o contrabando de sementes, plantações equivocadas e exploração predatória; a tentativa de obter sua síntese deu origem, mesmo que por desconhecimento, ao desenvolvimento da indústria química moderna e foi a base da indústria de corantes; sua síntese em 1944 foi feita (ainda que parcialmente) sem equipamentos de FT-RMN, coluna flash, CLAE ou T.L.C.;

Talvez a melhor maneira de encerrar essa história seja com o comentário de um dos principais atores que ainda está em atividade, ${ }^{23}$ Gilbert Stork: ${ }^{24}$ "O valor da síntese da quinina essencialmente não tem nada a ver com a quinina em si. É parecido com a resolução de um teorema matemático muito antigo, cuja solução foi procurada durante muito tempo. Amplia o conhecimento".

\section{AGRADECIMENTOS}

Ao Prof. A. Barisson pelas inúmeras revisões e discussões sobre o tema. Ao CNPq, Capes e Fundação Araucária pelo suporte financeiro.

\section{REFERÊNCIAS}

1. Brunton, L.; Lazo, J.; Parker, K.; Goodman and Gilman's the Pharmacological Basis of Therapeutics; 11 ${ }^{\text {th }}$ ed., McGraw-Hill: New York, 2006.

2. Le Couter, P.; Burreson, J.; Os Botões de Napoleão: As 17 moléculas que mudaram a história; trad. Borges, M.L.X. de A., Jorge Zahar ed.: Rio de Janeiro, 2006.

3. Os machos se alimentam de néctar e outras fontes de açúcar. Já as fêmeas, além dessa alimentação, necessitam se alimentar de sangue para o desenvolvimento dos ovos.

4. Pelletier, P. J.; Caventou, J. B.; Annales de Chimie et de Physique 1820, XV, 289.

5. Pasteur, L.; Comptes Rendus Hebdomadaires des Seances de l'Academie Sciences 1853, 37, 110.

6. Strecker, A.; Justus Liebig's Annalen der Chemie 1854, 91, 155, doi:10.1002/jlac.18540910204.

7. Garfield, S.; Mauve: How One Man Invented a Color that Changed the World, W. W. Norton \& Company: Scranton, 2001.

8. Para obter o corante, Perkin fabricou sua anilina pela rota da nitração do benzeno. Porém, na época, o benzeno era destilado do alcatrão de hulha (carvão mineral) e usualmente vinha contaminado com tolueno. Esta mistura foi submetida a uma reação de nitração $\left(\mathrm{HNO}_{3} / \mathrm{H}_{2} \mathrm{SO}_{4}\right) \mathrm{e}$ o produto obtido reduzido com ferro em ácido acético. Isto resultava em uma mistura de anilina, $o$ - e $p$ - toluidina, que não foi purificada 
mas colocada para reagir com um oxidante (dicromato de potássio), formando um corante púrpura.

9. Meth-Cohn, O.; Smith, S.; J. Chem. Soc., Perkin Trans. 1, 1994, 5, doi:10.1039/P19940000005.

10. Meth-Cohn e Smith receberam duas amostras, uma vinda da fábrica de Perkin da coleção de Perkin em Zeneca e a outra foi concedida pelo British Museum.

11. Seixas de Melo, J.; Takato, S.; Sousa, M.; Melo, M. J.; Parola, A.; Chem. Commun. 2007, 2624

12. Rabe, P.; Ackerman, E.; Schneider, W.; Berichte der Deutschen Chemischen Gesellschaft 1907, 40, 3655; Rabe, P.; Berichte der Deutschen Chemischen Gesellschaft 1908, 41, 62; Rabe, P.; Justus Liebig's Annalen der Chemie 1909, 365, 366, doi:10.1002/jlac.19093650214.

13. Carter, O. L.; McPhail, A. T.; Sim, O. A.; J. Chem. Soc. A 1967, 365, doi: 10.1039/j.1967.0000365.

14. Pasteur, L.; Comptes Rendus Hebdomadaires des Seances de l'Academie Sciences 1853, 37, 162.

15. Rabe, P.; Kindler, K.; Berichte der Deutschen Chemischen Gesellschaft 1939, 72, 263.
16. Woodward, R. B.; Doering, W. E.; J. Am. Chem. Soc. 1944, 66, 849, doi:10.1021/ja01233a516.

17. Woodward, R. B.; Doering, W. E.; J. Am. Chem. Soc. 1945, 67, 860, doi:10.1021/ja01221a051.

18. Stork, G.; Chem. Eng. News 2000, 78, 8 .

19. Stork, G.; Niu, D.; Fujimoto, A.; Koft, E. R.; Balkovec, J. M.; Tata, J. M.; Duke, G. R.; J. Am. Chem. Soc. 2001, 123, 3239, doi:10.1021/ ja004325r.

20. Seeman, J. I.; Angew. Chem., Int. Ed. 2007, 46, 1378, doi:10.1002/ anie. 200601551.

21. William von Eggers Doering é professor associado do Department of Chemistry and Chemical Biology na Harvard University.

22. Smith, A. C.; Williams, R. M.; Angew. Chem., Int. Ed. 2008, 47, 1736, doi:10.1002/anie.200705421.

23. http://pubs.acs.org/cen/coverstory/83/8325/8325quinine.html, acessada em Abril 2008.

24. Gilbert Stork é professor associado do Department of Chemistry na Columbia University (NY). 